\title{
Visita de estudios a Madrid en el Marco del Proyecto IRUDESCA
}

\section{Study visit to Madrid in the Project Framework IRUDESCA}

DOI: $\underline{\text { http://dx.doi.org/10.5377/uyc.v10i16.6141 }}$

\author{
Violeta Gago García \\ violeta2689@ hotmail.com \\ Universidad Nacional Autónoma de Nicaragua, Managua.
}

(C) UNAN-Managua

Recibido: octubre 2017. Aprobado: octubre 2017.

\section{RESUMEN}

La visita de estudio a Madrid fue la actividad final de una fase de "formación de 24 formadores en emprendimiento de 12 universidades de Centroamérica" contemplada en el marco del Proyecto IRUDESCA, que se inició en el mes de julio 2017 el cual fue liderado por la la Universidad Camilo José Cela (España), Escuela Superior de Comercio de París (ESCP Europe, sede Madrid) y Dinamia Sociedad Cooperativa (España). En la visita se conocieron nuevas formas de emprendimiento y estructuras empresariales, modelos de integración económica y cooperación empresarial europea. Este profesorado será el responsable de la formación a alumnos y Gerencias Asistidas posteriores. Para la UNAN-Managua fueron asignadas las Maestras, Misty Ramos y Violeta Gago.

Palabras clave: proyecto, empresariales, cooperación, formación, universidad.

\section{SUMMARY}

The study visit to Madrid was the final activity of a phase of "training 24 trainers in entrepreneurship of 12 universities in Central America" contemplated within the framework of the IRUDESCA Project, which began in July 2017, which was led by the the Camilo José Cela University (Spain), the Higher School of Commerce of Paris (ESCP Europe, Madrid headquarters) and Dinamia Cooperative Society (Spain). During the visit, new forms of entrepreneurial and business structures, models of economic integration and European business cooperation were known. This faculty will be responsible for the training of students and later Assisted Managers. For the UNANManagua were assigned the Teachers, Misty Ramos and Violeta Gago.

Keywords: project, business, cooperation, training, university.

La visita de estudio a Madrid fue la actividad final de una fase de "formación de 24 formadores en emprendimiento de 12 universidades de Centroamérica" contemplada en el marco del Proyecto IRUDESCA, que se inició en el mes de julio 2017 el cual fue liderado por la la Universidad Camilo José Cela (España), Escuela Superior de Comercio de París (ESCP Europe, sede Madrid) y Dinamia Sociedad Cooperativa (España). En la visita se conocieron nuevas formas de emprendimiento y estructuras empresariales, modelos de integración económica y cooperación empresarial europea. Este profesorado 


\section{universidâd}

será el responsable de la formación a alumnos y Gerencias Asistidas posteriores. Para la UNAN-Managua fueron asignadas las Maestras, Misty Ramos y Violeta Gago.

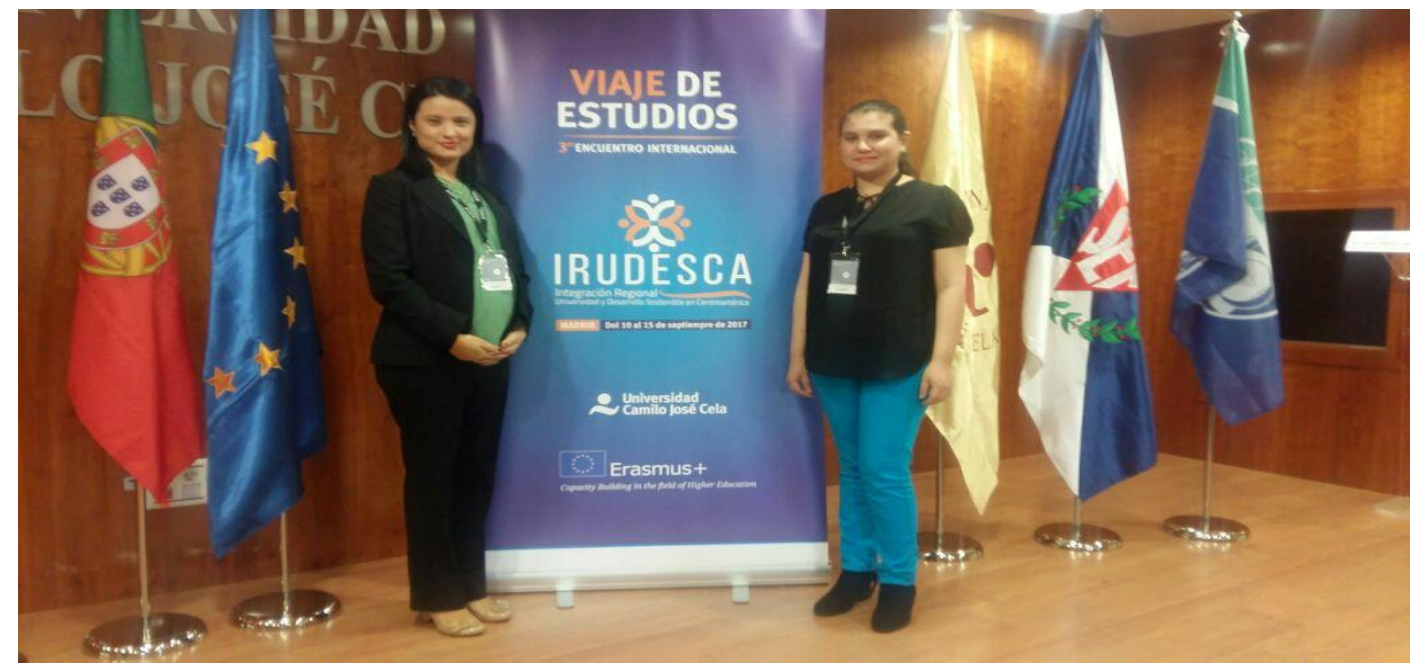

Figura 1. Maestra Misty Ramos y Violeta Gago en la visita de estudios en Madrid, Universidad Camilo José Cela (España).

Los resultados de la visita de estudio fueron:

$\checkmark 24$ profesores capacitados para formar en los módulos (Nuevos Contenidos Académicos del Proyecto IRUDESCA).

$\checkmark 24$ profesores habrán conocido estudios de caso de emprendimiento empresarial europeo, y habilidades de tutorización.

$\checkmark$ Organizarán la fase de formación y tutorización de los estudiantes.

$\underline{\text { Galería de fotos de la visita de estudio }}$

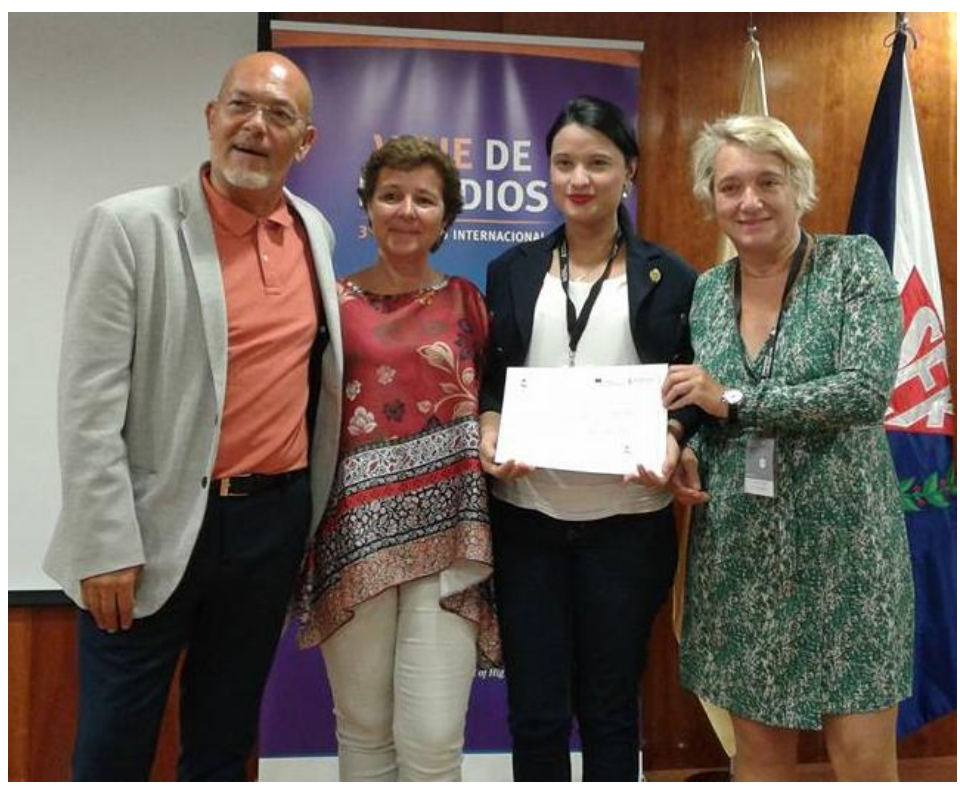

Figura 1. Helena Santos Coordinadora del Proyecto IRUDESCA, Lidio González Docente Universidad Camilo José Cela, Maestra Violeta Gago recibiendo diploma de culminación del curso formador de formadores en emprendimiento y Susana Méndez Docente Universidad Camilo José Cela.

Visita de estudios a Madrid en el marco de IRUDESCA... Violeta Gago, pp. 57- 61 
Visita de estudios a Madrid en el marco de IRUDESCA... Violeta Gago, pp. 57- 61

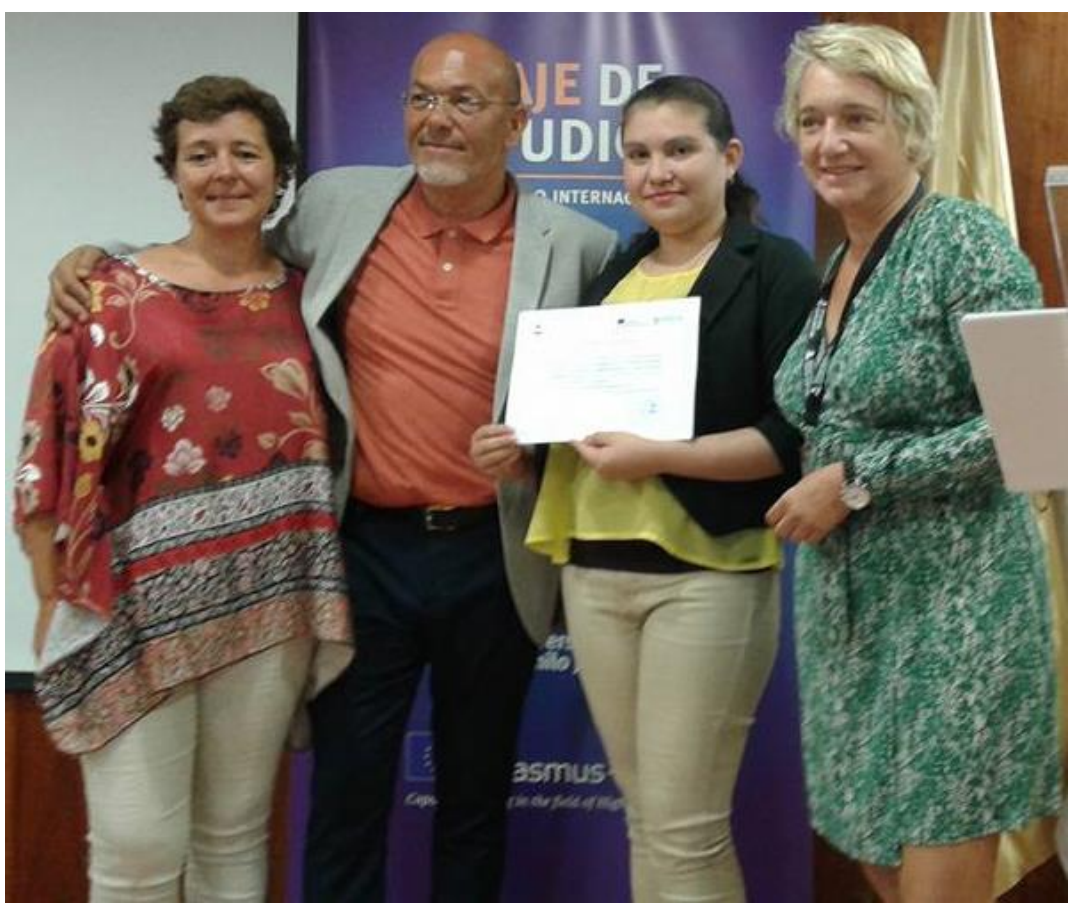

Figura 2. Lidio González Docente Universidad Camilo José Cela, Helena Santos Coordinadora del Proyecto IRUDESCA, Maestra Misty Ramos recibiendo diploma de culminación del curso formador de formadores en emprendimiento y Susana Méndez Docente Universidad Camilo José Cela.

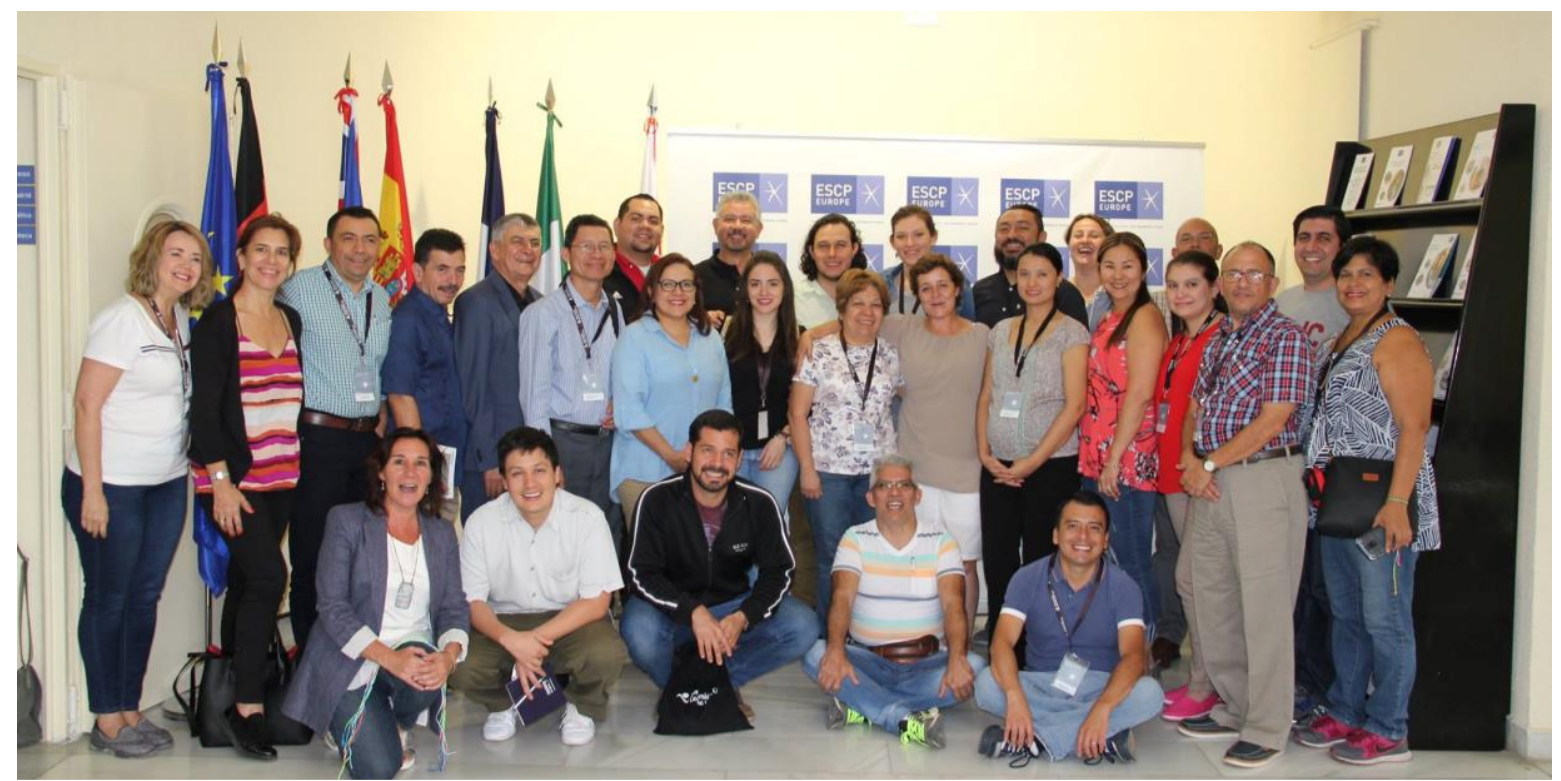

Figura 3. 24 profesores de Universidades de Centroamérica finalizando el curso formador de formadores en emprendimiento en la visita de estudios a Madrid en el marco del Proyecto IRUDESCA. Tercer encuentro internacional. Fuente. Escuela de Negocios de Europa ESCP Europe Madrid Campus. 


\section{universidâd}

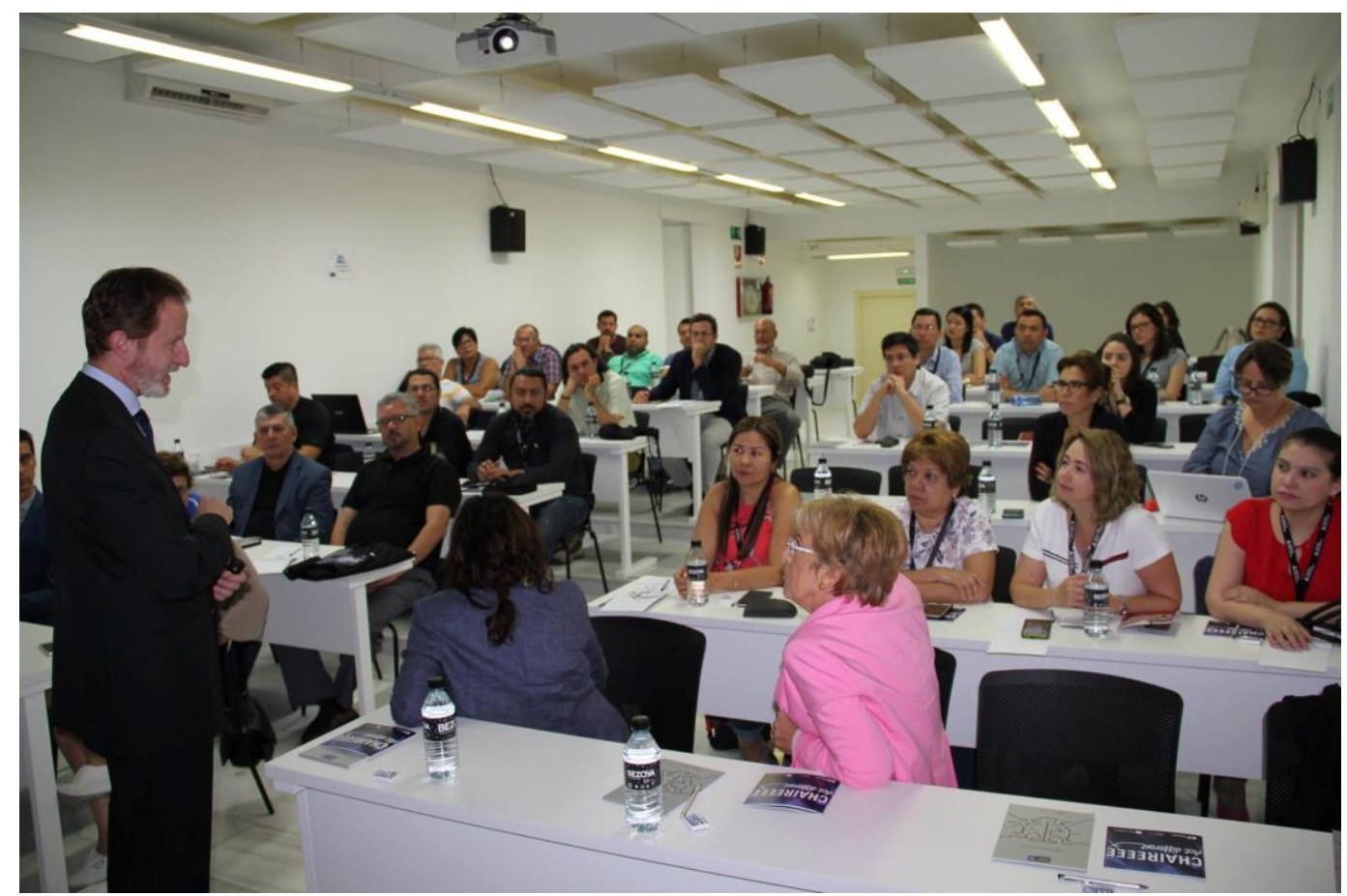

Figura 4. 24 profesores de Universidades de Centroamérica. Dr. Javier Tafur. Director del Campus de Madrid, Formando emprendedores: CHAIREEE. Cátedra. Modelo de referencia europeo: Multi Cultural-Multi Campus de referencia europea (Paris, Berlin, Londres, Turín, Varsovia). Fuente. Escuela de Negocios de Europa ESCP Europe Madrid Campus.

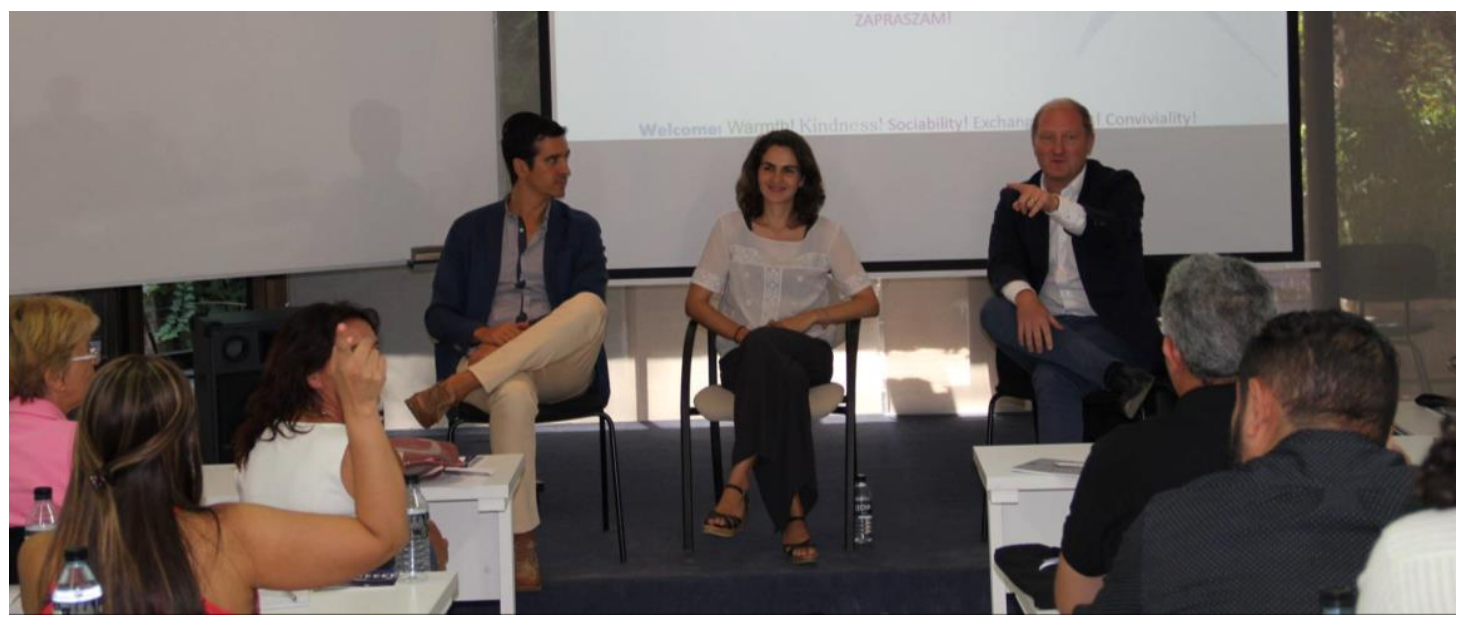

Figura 5. Tres buenas prácticas de emprendimiento: Emprendimiento en primera persona: $A\{2 H\} D E$ adoshorasde.org. Por Pedro Aizpun Alonso, CEO A\{2H\}DE y alumno ESCP Europe. Emprender y APLICAR El modelo SPRIM http://www.sprim.es/. Por Olivier Shleifer, Miembro Fundador y Managing Partner. Empresas con ánimo de CAMBIO/IMPACTO/DISEÑO/INNOVACIÓN SOCIAL: KOAN http://www.koanconsulting.com/ ISANNAS http://www.sannas.eu/: Asociación empresarial por el triple balance. Marina Cruz, Responsable Gestión de Proyectos Fuente. Escuela de Negocios de Europa ESCP Europe Madrid Campus.

Visita de estudios a Madrid en el marco de IRUDESCA... Violeta Gago, pp. 57- 61 